\title{
Identification and Analysis of Putative Polyhydroxyalkanoate Synthase (PhaC) in Pseudomonas fluorescens ${ }^{\text {[S }}$
}

\author{
Ju Hyoung Lim ${ }^{1,2}$, Ho-Gun Rhie ${ }^{3}$, and Jeong Nam Kim ${ }^{4,5 *}$ \\ ${ }^{1}$ Department of Biological Sciences, Korea Advanced Institute of Science and Technology (KAIST), Daejeon 34141, Republic of Korea \\ ${ }^{2}$ Department of Civil and Environmental Engineering, Massachusetts Institute of Technology, Cambridge, MA 02142, USA \\ ${ }^{3}$ Department of Biology, Kyung Hee University, Seoul 02447, Republic of Korea \\ ${ }^{4}$ Department of Microbiology, College of Natural Sciences, Pusan National University, Busan 46241, Republic of Korea \\ ${ }^{5}$ Department of Integrated Biological Science, College of Natural Sciences, Pusan National University, Busan 46241, Republic of Korea
}

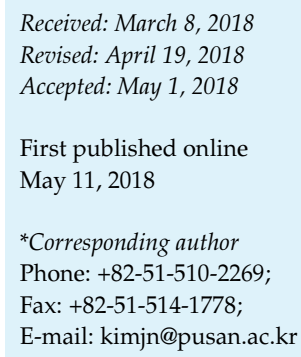

$S$ upplementary data for this paper are available on-line only at http://jmb.or.kr.

pISSN 1017-7825, eISSN 1738-8872

Copyright (C) 2018 by

The Korean Society for Microbiology and Biotechnology
Pseudomonas fluorescens KLR101 was found to be capable of producing polyhydroxyalkanoate (PHA) using various sugars and fatty acids with carbon numbers ranging from 2 to 6 . The PHA granules consisted mainly of a poly(3-hydroxybutyrate) homopolymer and/or poly(3hydroxybutyrate-co-3-hydroxyvalerate) copolymer. Genomic DNA of P. fluorescens was fractionated and cloned into a lambda library, in which a 5.8-kb fragment that hybridized to a heterologous phaC probe from Ralstonia eutropha was identified. In vivo expression in Klebsiella aerogenes KC2671 (pUMS), restriction mapping, Southern hybridization experiments, and sequencing data revealed that PHA biosynthesis by $P$. fluorescens relied upon a polypeptide encoded by a 1,683-bp non-operonal ORF, which was preceded by a possible $-24 /-12$ promoter and highly similar to DNA sequences of a gene encoding PHA synthase in the genus Pseudomonas. In vivo expression of the putative PHA synthase gene $\left(p h a C_{\mathrm{Pf}}\right)$ in a recombinant Escherichia coli strain was investigated by using glucose and decanoate as substrates. E. coli $\left(\mathrm{phaC}_{\mathrm{Pf}}{ }^{+}, \mathrm{pUMS}\right)$ grown in medium containing glucose accumulated PHA granules consisting mainly of 3-hydroxybutyrate, whereas only a trace amount of 3-hydroxydecanoate was detected from an E. coli fadR mutant $\left(p h a C_{\mathrm{Pf}}{ }^{+}\right)$grown in medium containing decanoate. In vitro enzymatic assessment experiments showed that 3-hydroxybutyryl-CoA was efficiently used as a substrate of purified $\mathrm{PhaC}_{P f}$, suggesting that the putative PHA synthase of $P$. fluorescens utilizes mainly short-chain-length PHA precursors as a substrate.

Keywords: Pseudomonas fluorescens KLR101, polyhydroxyalkanoate (PHA), putative PHA synthase gene, short-chain-length PHA precursors

\section{Introduction}

Polyhydroxyalkanoate (PHA) is synthesized and accumulated as insoluble inclusions by various bacteria, when cells are cultivated in the presence of excess carbon sources along with a shortage of essential nutrients such as nitrogen, phosphate, oxygen, and/or sulfur ions [1]. PHA has received considerable interest as a biodegradable and biocompatible plastic with a wide range of potential applications [2]. Since the cloning of the PHA synthase operon of Ralstonia eutropha [3-5], numerous genes from a wide range of bacteria have been cloned or identified [6, 7]. A total of 338 different PHA synthases from 214 species are available via Entrez protein search at the NCBI website (http://www.ncbi.nlm.nih.gov/). PHA synthases identified from members of the genus Pseudomonas contribute to the largest proportion ( $23 \%$ of total), which includes the recently identified phaC loci from two $P$. fluorescens strains obtained by full genome sequencing (GenBank No. CP000076 and CP000094). Likewise, accumulated evidence indicates that $P$. fluorescens strains are capable of PHA production [8-12]. However, their genetic backgrounds 
have never been functionally evaluated. Prior investigations into the capacity of PHA accumulation by $P$. fluorescens KLR101 have reported the detection of poly(3hydroxybutyrate) homopolymers and poly(3-hydroxybutyrateco-3-hydroxyvalerate) copolymers from cells grown in the presence of various carbon sources. A genomic fragment responsible for PHA production was obtained from a lambda library, and a PHA synthase gene (phaC) was identified by cloning, in vivo expression tests, and in vitro enzymatic assays.

\section{Materials and Methods}

\section{Bacterial Strains and Plasmids}

The bacterial strains and plasmids used in this study are listed in Table 1. The Pseudomonas fluorescens and Klebsiella aerogenes strains were routinely propagated on nutrient agar at $28^{\circ} \mathrm{C}$. The Escherichia coli strains were grown in LB medium at $37^{\circ} \mathrm{C}$, and $100 \mu \mathrm{g} / \mathrm{ml}$ of ampicillin, $50 \mu \mathrm{g} / \mathrm{ml}$ of kanamycin, $50 \mu \mathrm{g} / \mathrm{ml}$ of chloramphenicol, and/or $15 \mu \mathrm{g} / \mathrm{ml}$ of tetracycline were added to the media, as indicated in Table 1.

\section{DNA Manipulation and Sequence Analyses}

Primers I-179L and I-179R were used for PCR detection of the 540-bp phaC region [10]. Nucleotide sequencing analysis of the plasmids was performed using vector-borne primers or sequencebased internal primers. The nucleotide and deduced amino acid sequences for PHA synthase of $P$. fluorescens KLR101 were compared with all sequences available in the GenBank database and aligned and edited using CLUSTAL_X [13] and BIOEDIT software (http:// www.mbio.ncsu.edu/bioedit/bioedit.html). Phylogenetic trees were constructed using MEGA 3.1 software [14]. The GenBank/ EMBL/DDBJ accession number for the newly determined phaC gene sequence of $P$. fluorescens is AY232443.

\section{Plasmid Construction}

A genomic DNA library of $P$. fluorescens KLR101 was constructed using a Lambda DASH kit (Stratagene, USA). Plaque hybridization was performed with the 573-bp PstI fragment of the phbC gene from Ralstonia eutropha [15] by using a Genius kit (Boehringer Mannheim, USA). A 5.8-kb BglII fragment showing a positive signal was then cloned into the BamHI site of pBluescript II SK+ (Stratagene, USA). The resulting plasmid was used to transform Klebsiella aerogenes KC2671 (pUMS) [15] and then tested for in vivo expression. A 3.8-kb EcoRI fragment was then subcloned into pBluescript II SK+. The entire coding region for PHA synthase in P. fluorescens KLR101 was amplified from the 3.8-kb EcoRI fragment using forward primers (5'-GGAGTGTTGGCATATGCGAGAAAG ACC-3' for pT7-7CPf and 5'-GGAGTGTGGATCCATGCGAGA AAGACC- $3^{\prime}$ for pRSET-ACPf) containing NdeI and BamHI enzyme sites, respectively (underlined), and reverse primers (5'-TGATCGCATATGTCAGCGCACTCGCA-3' for pT7-7CPf and 5'-TGATCGCCATGGTCAGCGCACTCGCA-3' for pRSET-ACPf) containing NdeI and NcoI enzyme sites, respectively (underlined). The amplified products were digested using the corresponding

Table 1. Bacterial strains and plasmids used in this study.

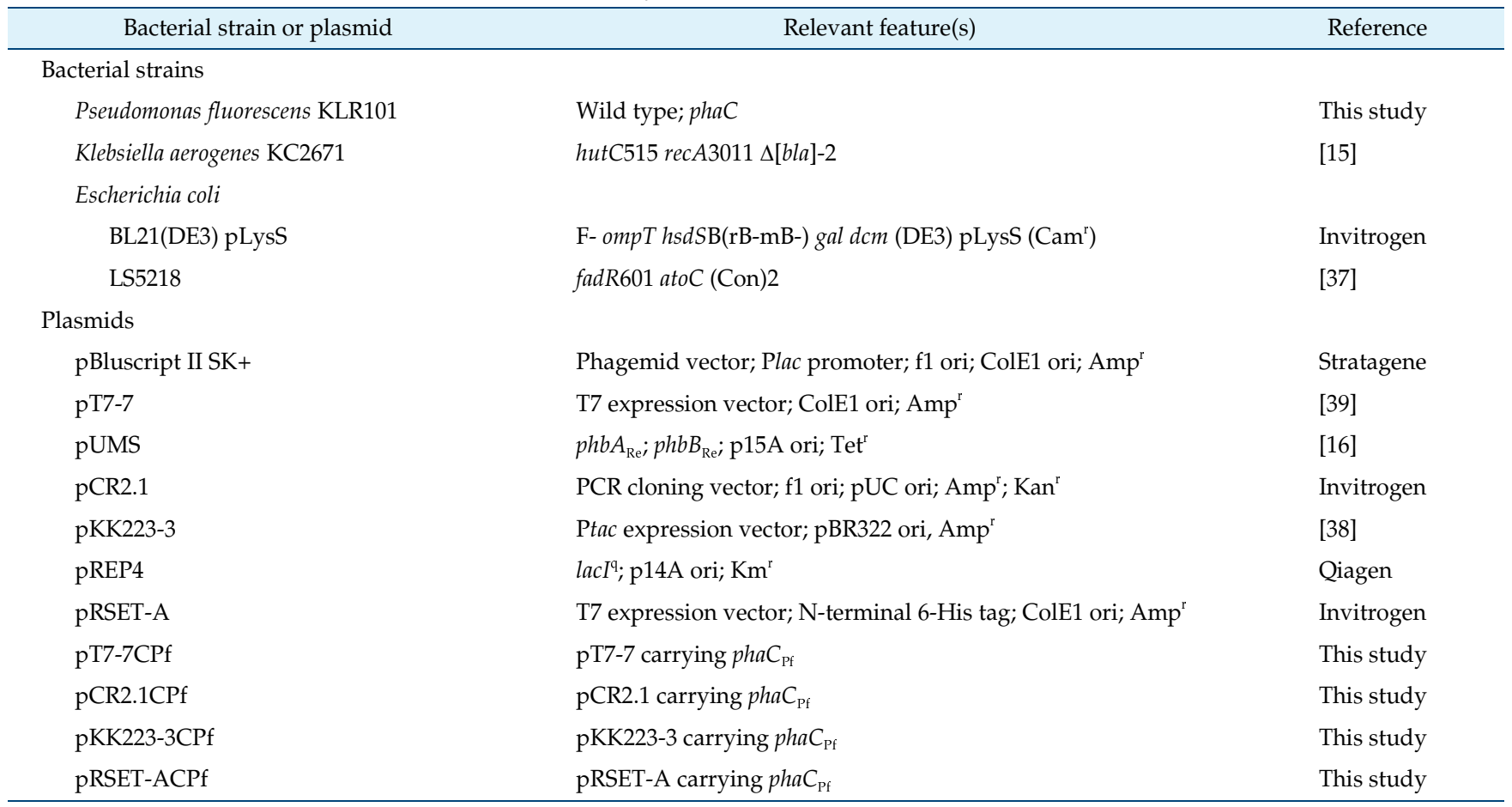


enzymes and ligated to polylinker regions of $\mathrm{T} 7$ expression vectors, pT7-7 and pRSET-A, resulting in pT7-7CPf and pRSETACPf, respectively. An AvaI site located 570 bp downstream from the start codon was used to confirm the correct direction of the phaC ORF on pT7-7CPf. Polylinker regions of the recombinant plasmids were sequenced for confirmation of in-frame cloning. Plasmids pT7-7CPf and pRSET-ACPf were then transformed into E. coli BL21 (DE3) pLysS. After the overexpression of the appropriate polypeptide was confirmed by SDS-PAGE experiments, E. coli BL21(DE3) pLysS (pT7-7CPf) was transformed using pUMS [16] for the in vivo expression test.

Plasmid pKK223-3CPf was constructed as follows. The ORF for PHA synthase was amplified using forward (5'-ATGCGAGAA AGACCCGTGACG-3') and reverse (5'-TCAGCGCACTCGCAC ATAGGT-3') primers, and cloned into the pCR2.1 vector (Invitrogen, USA), from which a 1.7-kb EcoRI fragment containing the phaC ORF was excised and cloned into the tac expression vector pKK223-3. In-frame cloning was confirmed by sequencing of a polylinker region. The plasmid pKK223-3CPf was then used to transform E. coli LS5218, which was subsequently transformed by the LacI expression vector pREP4 (Qiagen, USA). Overexpression of putative PhaC in the strain LS5218 (pKK223-3CPf and pREP4) by IPTG induction was confirmed by SDS-PAGE experiments. Plasmid maps were drawn using CloneMap software (CGC Scientific, USA).

\section{Overexpression of PHA Synthase}

Overnight cultures of recombinant $E$. coli strains were harvested and washed, and a portion of the cell suspension was inoculated into $100 \mathrm{ml}$ of freshly prepared of LB medium. Protein production was induced by the addition of IPTG $(0.4 \mathrm{mM})$ for $2 \mathrm{~h}$, followed by purification and separation on a 10\% SDS-PAGE gel using the CBS Lite slab-gel system (CBS Scientific, USA). Protein bands were visualized by staining using Coomassie brilliant blue R-250 (BioRad, USA), and the gels were dried using gel-drying films (Promega, USA).

\section{In Vivo Expression of PHA Synthase and Detection of PHA}

$P$. fluorescens and recombinant $K$. aerogenes were cultivated in a reciprocal shaker for $72 \mathrm{~h}$ in standard nutrient liquid (SNL) medium [17] supplemented with carbon sources. E. coli BL21(DE3) pLysS (pT7-7CPf and pUMS) was incubated in LB medium containing $1 \%(\mathrm{w} / \mathrm{v})$ glucose for 48 h. E. coli LS5218 (pKK223$3 \mathrm{CPf}$ and pREP4) was incubated in LB medium containing $0.2 \%$ $(\mathrm{w} / \mathrm{v})$ decanoate in the presence of $0.2 \mathrm{mg} / \mathrm{ml}$ of acrylate [18], to which glucose $(1 \%(\mathrm{w} / \mathrm{v}))$ was added for cell growth. A stock solution of decanoate was prepared by dissolving in $0.5 \%(\mathrm{w} / \mathrm{v})$ Brij58 [19]. IPTG was added to LB medium at a concentration of $0.2 \mathrm{mM}$ every $12 \mathrm{~h}$. PHA granules were selectively stained with Nile blue A (Sigma, USA) according to Ostle and Holt [20] and examined under a confocal microscope. Cells were harvested, washed, lyophilized, and subjected to methanolysis according to Brandl et al. [21]. Gas chromatographic analysis was performed using an HP-Innowax crosslinked PEG column $(60 \mathrm{~m} \times 0.32 \mathrm{~mm} \times$ $0.25 \mu \mathrm{m}$ ) with the HP 6890 GC system (Agilent, USA).

\section{In Vitro Enzymatic Assessment of Soluble PHA Synthase}

The enzymatic activity of soluble PHA synthase was determined using either purified enzyme or crude extracts. Histagged protein was purified from an overexpressed cell culture of E. coli BL21(DE3) pLysS (pRSET-ACPf) using the ProBond purification system (Invitrogen, USA), in which a purified fraction was concentrated and reconstituted with KPi buffer (143 mM KPi, $\mathrm{pH} 7.2$, and $5 \%(\mathrm{v} / \mathrm{v})$ glycerol) using a 30,000 MW cutoff filter, Centricon YM-30 (Merk, USA). A portion of the purified fraction was examined by SDS-PAGE. Soluble cell extracts of E.coli BL21(DE3) pLysS (pT7-7CPf) were prepared according to Qi et al. [22]. The enzyme assay was performed based on the off-line Ellman method [22-26] using $0.5 \mathrm{mM}$ DL- $\beta$-hydroxybutyryl-CoA (Sigma, USA) as a substrate. The relative concentration of free thiols was calculated according to the equation $C_{o}=A / \varepsilon \times D$ [9], where $C_{o}$ is the original concentration of CoA thioester, $A$ is the absorbance at $412 \mathrm{~nm}, \varepsilon$ is the extinction coefficient $(13.7 \mathrm{mM} / \mathrm{cm})$, and $D$ is the dilution factor. Nonspecific hydrolysis of $\mathrm{CoA}$ thioester was monitored using a negative control.

\section{Results and Discussion}

\section{Available Carbon Sources for PHA Production by P. fluorescens KLR101}

P. fluorescens KLR101 was capable of accumulating PHA granules when cells were grown in SNL medium

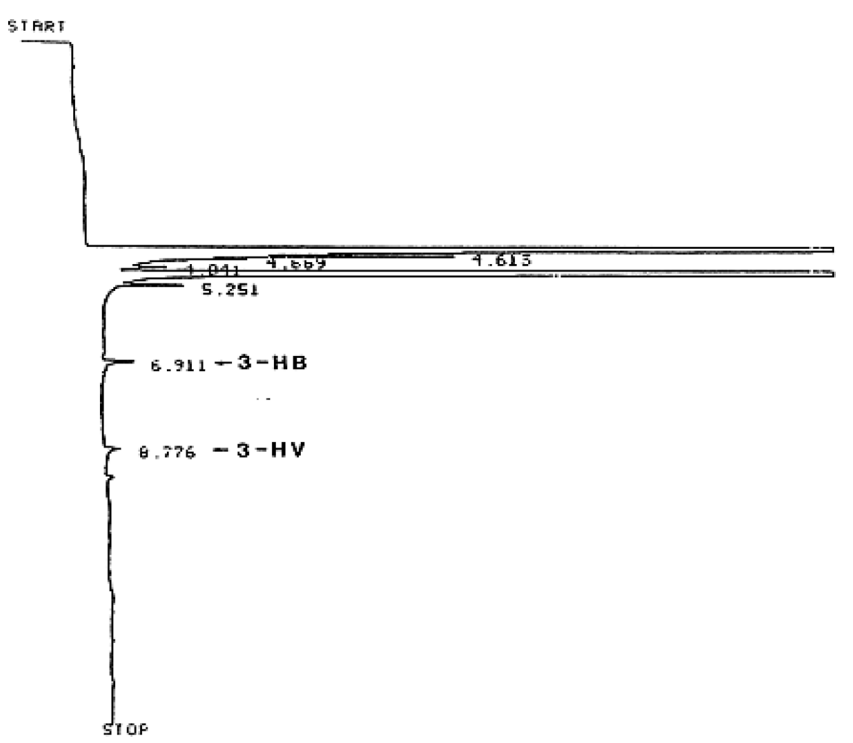

Fig. 1. Gas chromatogram of Pseudomonas fluorescens grown in $1 \%(\mathrm{w} / \mathrm{v})$ glucose and $10 \mathrm{mM}$ propionate.

3-HB and 3-HV indicate 3-hydroxybutyrate and 3-hydxroxyvalerate, respectively. 
A

\section{8 kb Lambda clone}

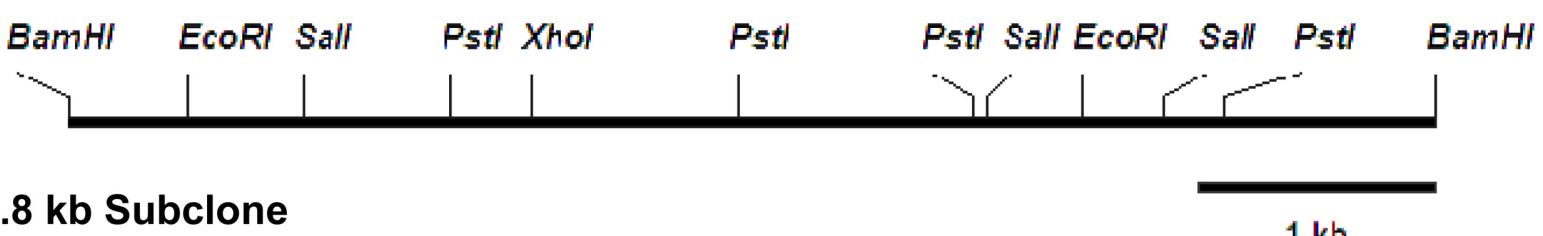

\section{8 kb Subclone}

\section{$1 \mathrm{~kb}$}

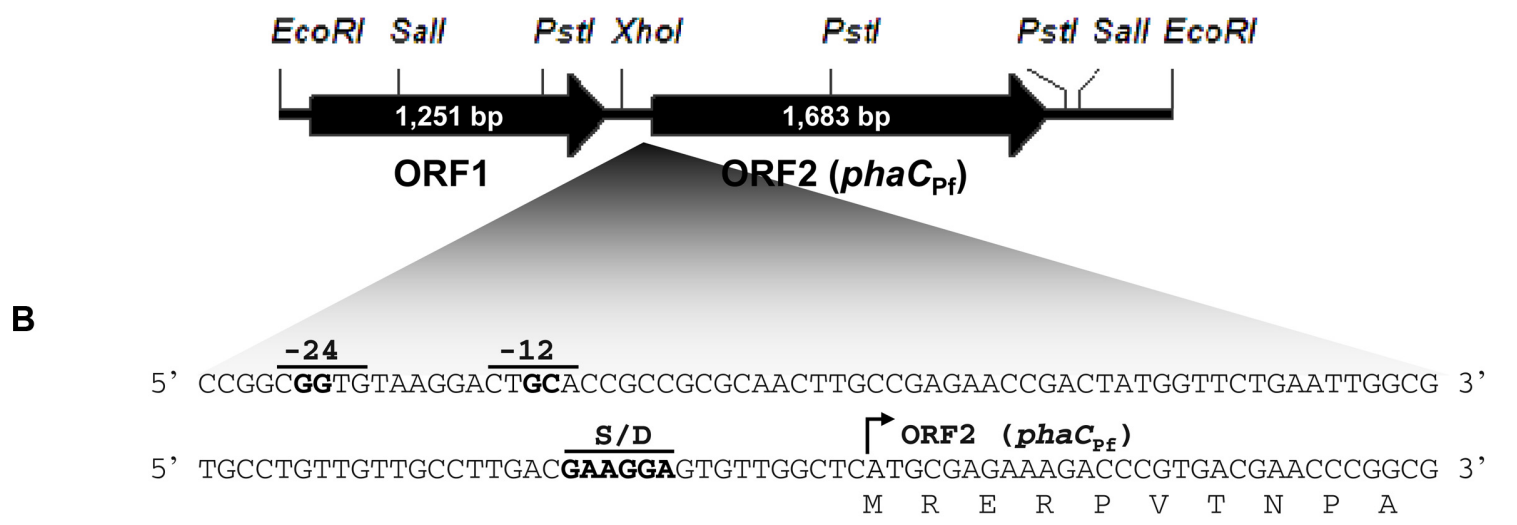

Fig. 2. Restriction maps of the clones harboring $p h a C_{\mathrm{Pf}}(\mathbf{A})$ and the $5^{\prime}$ UTR sequence of ORF2 (B).

A possible -24/-23 promoter and a Shine-Dalgarno (S/D) sequence are represented on the $5^{\prime}$ UTR sequence of ORF2 (B).

supplemented with various carbon sources, such as glucose $(1 \%(\mathrm{w} / \mathrm{v}))$, sucrose $(1 \%(\mathrm{w} / \mathrm{v}))$, sodium acetate $(0.1 \%(\mathrm{w} / \mathrm{v}))$, propionate $(10 \mathrm{mM})$, gluconate $(1 \%(\mathrm{w} / \mathrm{v}))$, sodium citrate $(0.1 \%(\mathrm{w} / \mathrm{v}))$, or benzoate $(5 \mathrm{mM})$. Gas chromatographic analyses showed that the predominant monomer unit was 3-hydroxybutryate. When the strain was grown in $1 \%(\mathrm{w} / \mathrm{v})$ glucose and $10 \mathrm{mM}$ propionate, production of a poly(3-hydroxybutyrate-co-3-hydroxyvalerate) copolymer was also observed (Fig. 1). Confirmation that P. fluorescens KLR101 possesses an intrinsic PHA biosynthesis pathway was carried out by amplification and sequencing analysis of the 540-bp internal region of the phaC gene encoding PHA synthase [10] (data not shown). Members of the genus Pseudomonas are well-known medium-chainlength (mcl)-PHA producers [27]. However, the profile of utilizable carbon sources revealed that the in vivo substrate range of strain KLR101 was mainly short-chain-length (scl)-PHA precursors.

\section{Genetic Organization of Two ORFs}

A 5.8-kb genomic fragment of the strain was identified from the lambda clone library using the heterologous gene probe from R. eutropha [15] (Fig. S1). As shown in Fig. 2A, the phaC gene was detected in a 3.8-kb EcoRI fragment, which was subcloned and sequenced (GenBank No. AY232443). A sequence similarity search was conducted, and two possible ORFs of approximately 1.25 and $1.68 \mathrm{~kb}$ in the $3.8-$ $\mathrm{kb}$ fragment were detected, which was consistent with the restriction mapping results (Fig. 2A). Of particular note, ORF2 was markedly matched with the phaC2 gene of Pseudomonas sp. KBOS 04 (GenBank No. AY790328) with $88 \%$ sequence similarity. Moreover, a potential ribosomebinding site [28] and possible $-24 /-12$ promoter region were identified in ORF2 [29, 30] (Fig. 2B). ORF1 consisted of $1,251 \mathrm{bp}$ with its own promoter (possible $-35 /-10$ promoter), and the deduced product was highly similar to the amino acid sequence of PhhR, a transcriptional activator of the phenylalanine hydroxylase gene cluster [31]. Despite the high sequence similarity with type II PHA synthase, the arrangement of the putative PHA synthase gene was slightly different from those of typical type II synthase genes. The phaC2 genes are hitherto organized as an operonal arrangement known as phaC1ZC2 [30, 32-35], of which gene expression is generally controlled by the $-24 /-12$ and/or $-35 /-10$ promoter preceding phaC1 [7, 30, 33]. Some phaC2 genes have been reported to have their own $-35 /-10$ promoters [33, 35], which is considered as bicistronicity, offering flexibility in response to environmental 
A

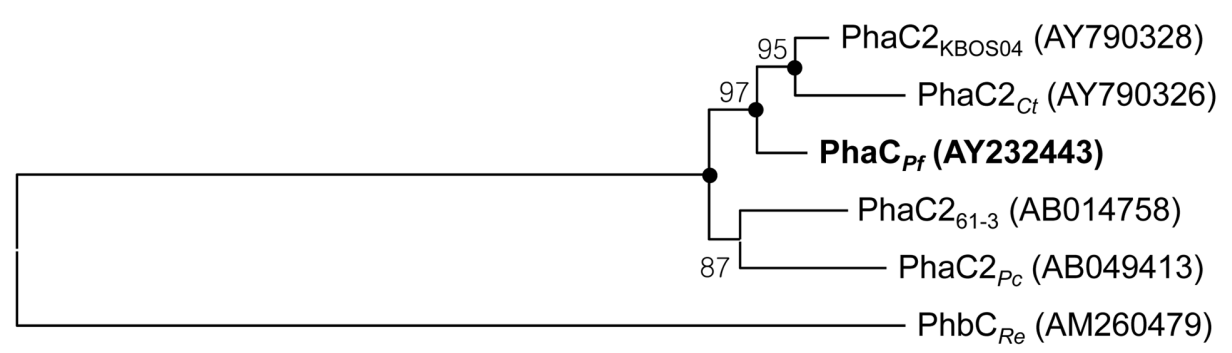

B

PhaC $_{P f}$
PhaC2 $_{\text {KBOS04 }}$
PhaC2 $_{61-3}$
PhaC2 $_{P c}$
PhaC2 $_{C t}$
PhbC $_{R e}$
PhaC $_{P f}$
PhaC2 $_{\text {KBOS04 }}$
PhaC2 $_{61-3}$
PhaC2 $_{P c}$
PhaC2 $_{C t}$
PhbC $_{R e}$

0.1

Ser ${ }^{238}$

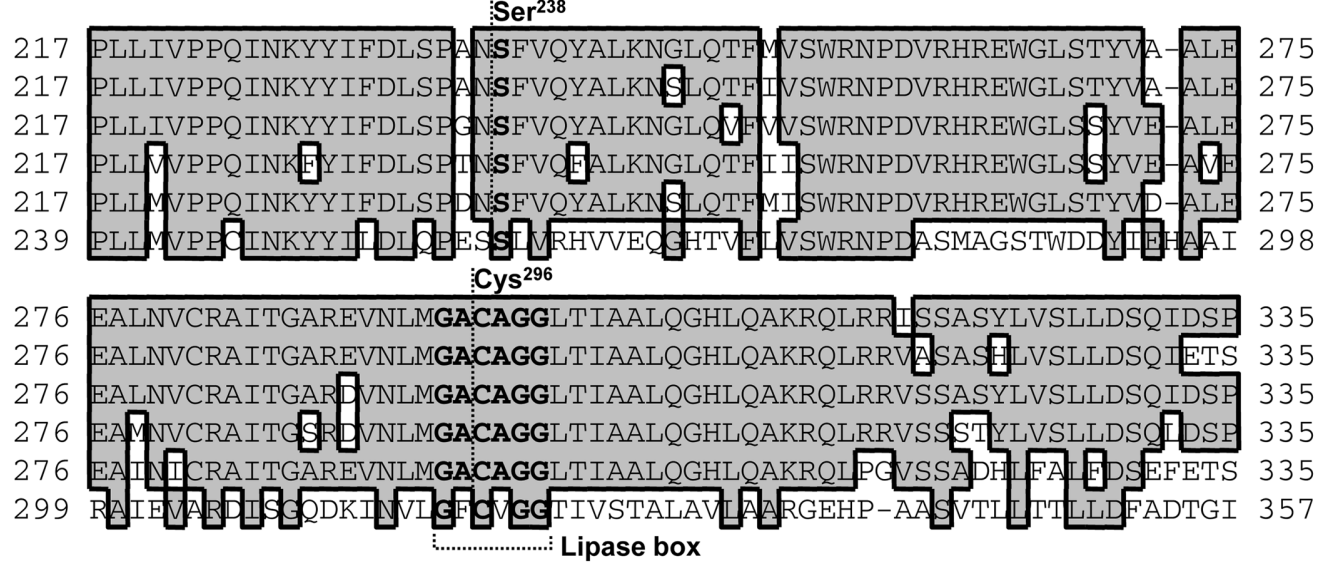

Fig 3. Phylogenetic relationship between the PHA synthase from P. fluorescens KLR101 and those from closely related strains. (A) Bootstrap values (using 1,000 replications) are indicated at the branch points. Filled circles demonstrate that the corresponding nodes were also recovered in maximum-parsimony trees [40]. Accession numbers are shown in parentheses. Bar, 0.1 expected nucleotide substitutions. (B) Partial sequence alignment is shown. Identical amino acids are shaded, and highly conserved motifs are indicated. PhaC $C_{P f}$, PhaC from $P$.

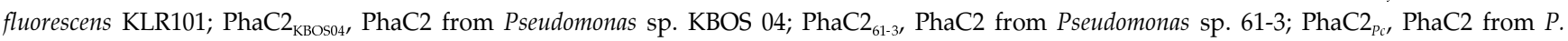
chlororaphis IFO 3521; PhaC2 ${ }_{C}$, PhaC2 from Comamonas testosteroni KBOS 01; $\mathrm{PhbC}_{R e}$, PhbC from Ralstonia eutropha H16.

changes. In order to determine whether or not ORF2 is a gene for PHA production, a functional expression system using the $K$. aerogenes KC2671 (pUMS) strain, which is unable to produce PHA, was employed. When in vivo expression of the 5.8-kb lambda fragment was conducted in this strain, PHB granule formation was only observed under nitrogen-limiting conditions (Fig. S2A), indicating that the $-24 /-12$ promoter preceding ORF2 could actively respond to environmental changes. Thus, the putative phaC gene is considered as a non-operonal gene and may have specific effects on PHA production in strain KLR101.

\section{Phylogenetic Analysis and Conserved Residues of Putative PhaC $_{P f}$}

A polypeptide, designated as $\mathrm{PhaC}_{P f}$, consisting of 560 amino acids with an isoelectric point of 9.12 and a calculated MW of $62.6 \mathrm{kDa}$, was deduced from ORF2, which is in agreement with well-known properties of PHA synthases [2]. In a phylogenetic tree constructed by the neighbor-joining algorithm [36], $\mathrm{PhaC}_{P f}$ formed a coherent cluster with PHA synthases identified from members of the genera Pseudomonas and Comamonas (Fig. 3A). Functionally important residues for enzymatic activity were conserved in the lipase box (Gly-X-Cys $\left.{ }^{296}-X-G l y-G l y\right) ~[2,30]$, in which $\mathrm{Cys}^{296}$ is known as a catalytic thiol covalently bonded to a growing polymer chain [25] (Fig. 3B). The possible aspartate and histidine residues that participate in the catalytic triad were identified as $\mathrm{Asp}^{329}, \mathrm{Asp}^{401}$, $\mathrm{His}^{453}$, and $\mathrm{His}^{480}$ (data not shown). Highly conserved Ser ${ }^{238}$, which is known as a target of post-translational 4-phosphopanthetheinylation [25], was also detected in the primary structure of $\mathrm{PhaC}_{P f}$ (Fig. 3B).

\section{Overexpression and Functional Expression of Putative PhaC $_{P f}$}

The entire region of ORF2 was amplified using NdeI-site 


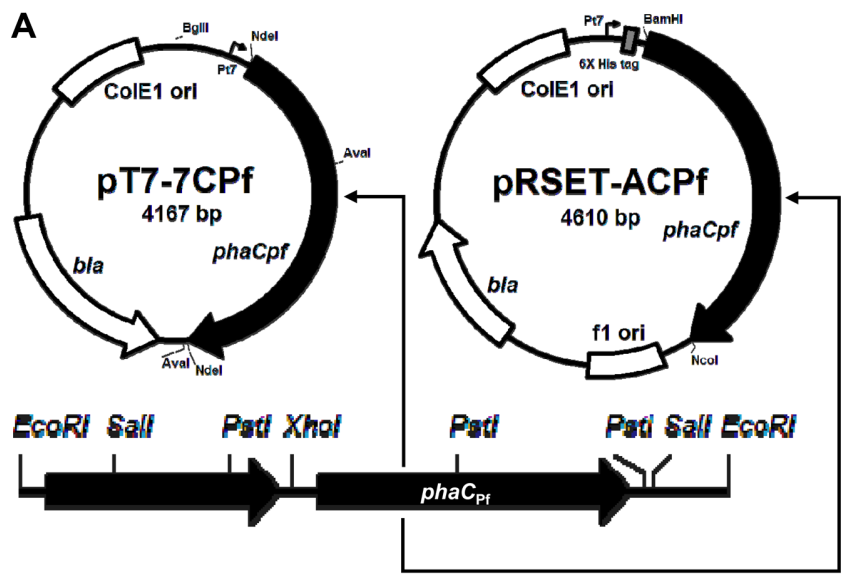

B

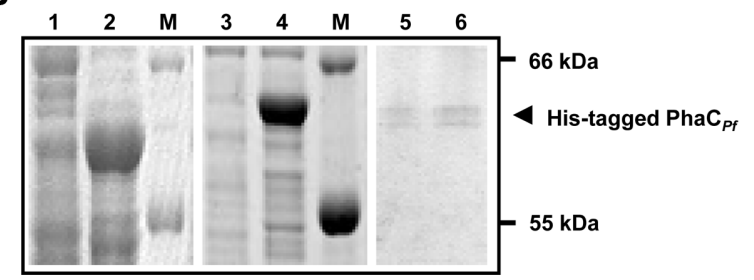

Fig. 4. Plasmid construction and overexpression of $\mathrm{PhaC}_{P f}$. (A) ORF2 was cloned non-directionally and directionally into pT7-7 and pRSET-A, respectively, via tailed PCR cloning. AvaI sites, which were used as orientation markers, are indicated on pT7-7CPf. (B) Overexpression of the two plasmids was visualized on a $10 \%$ SDSPAGE gel. Lanes 1 and 2, crude lysates of pT7-7CPf clones without and with IPTG, respectively; lanes 3 and 4 , crude lysates of pRSETACPf clones without and with IPTG, respectively; lanes 5 and 6, 10 and $20-\mu \mathrm{l}$ portions of the purified fraction of pRSET-ACPf-clone lysate, respectively; M, Mark12 Unstained standard (Invitrogen, USA).

containing primers and cloned into expression vector pT77 , resulting in pT7-7CPf (Fig. 4A). Using sequencing, the start codon of ORF2 was confirmed to be preceded by a ribosome-binding site and a $\mathrm{T} 7$ promoter without an internal start codon. Overexpression test of pT7-7CPf in E. coli BL21(DE3) pLysS showed that a polypeptide of around $60 \mathrm{kDa}$ was overproduced upon IPTG induction (Fig. 4B, lanes 1 and 2). Recombinant E. coli was then transformed using the plasmid pUMS harboring phbA (3ketothiolase gene) and $p h b B$ (acetoacetyl-CoA reductase gene). Cells grown in $1 \%(\mathrm{w} / \mathrm{v})$ glucose and $0.4 \mathrm{mM}$ IPTG accumulated PHA granules that consisted mainly of 3hydroxybutyrate (Fig. S2), indicating that PHA synthase from P. fluorescens KLR101 was functionally expressed under in vivo conditions. Decanoate as a substrate was also tested using the E. coli fadR atoC (Con) mutant LS5218 [37].

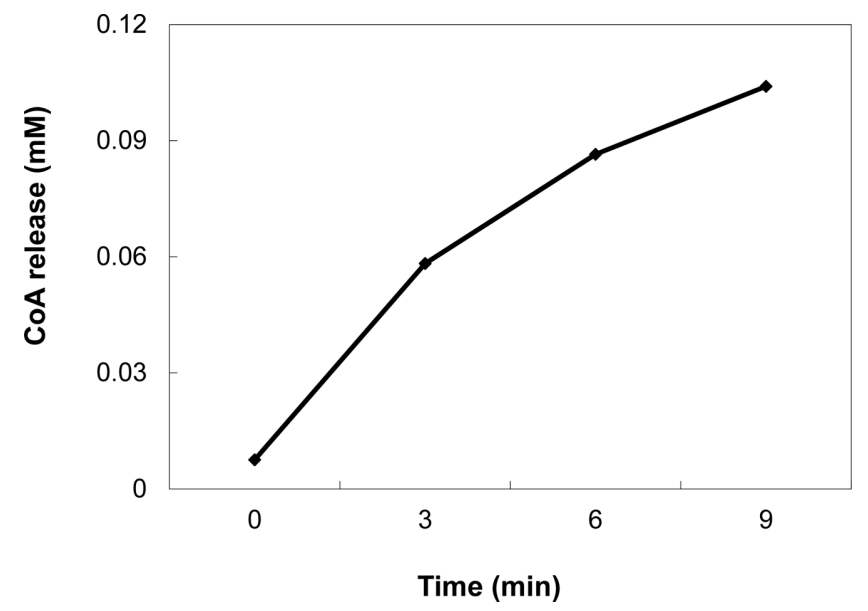

Fig. 5. Reaction kinetics of His-tagged $\mathrm{PhaC}_{P f}$ for 3-HB-CoAs. The reaction was initiated by addition of substrate, and the time course of CoA release was monitored at $412 \mathrm{~nm}$ with 5,5'-dithiobis-2nitrobenzoic acid [23].

To reduce the possibility that the $\beta$-oxidation pathway was not fully activated in the fatty acid-adapted cell portion, E. coli LS5218 was employed as a host strain. ORF2 was cloned into a tac promoter vector, pKK223-3 [38], and the resulting plasmid pKK223-3CPf was transformed into strain LS5218, which was then co-transformed with pREP4 as the IPTG control. Recombinant E. coli showed an overexpression profile similar to that of E. coli BL21(DE3) pLysS (pT7-7CPf) (data not shown). The strain was cultivated with $0.2 \%(\mathrm{w} / \mathrm{v})$ decanoate in the presence of $1 \%$ $(\mathrm{w} / \mathrm{v})$ glucose for cell growth and mcl-PHA production was induced by the addition of acrylate [18]. However, only a trace amount of 3-hydroxydecanoate was detected by GC analysis (Fig. S2B).

To demonstrate the in vitro enzymatic activity of soluble $\mathrm{PhaC}_{P f}$ ORF2 was cloned into pRSET-A (Fig. 4A). Sequencing of a polylinker region of the resulting plasmid (pRSETACPf) confirmed that a polypeptide translated from the plasmid was in-frame with a leader peptide (36 amino acids) containing a hexahistidyl sequence, which was consistent with the overexpression profile of pRSET-ACPf (Fig. 4B, lanes 3 and 4). His-tagged $P_{P h C}$ was then purified and visualized on a SDS-PAGE gel (Fig. 4B, lanes 5 and 6). The purified protein was used for enzymatic assays as follows.

\section{Enzymatic Activity of the Putative PHA Synthase}

The reaction kinetics of the purified enzyme with 3hydroxybutyryl-CoA over a short period demonstrated a proportional relationship (Fig. 5), indicating a high 
substrate preference of His-tagged $\mathrm{PhaC}_{P f}$ for the scl-PHA monomer. The soluble cell lysate of E. coli BL21(DE3) pLysS (pT7-7CPf) showed similar reaction kinetics (data not shown), which indicates no difference in enzyme activity between the wild-type and fusion proteins. Thus, it appears that polymerization was initiated immediately after substrate addition without any lag period, and the reaction was sufficiently efficient to release free CoA thioesters at a rate of $10.7 \mu \mathrm{M} / \mathrm{min}$.

Taken together, the phylogenetic analyses indicate that the putative PHA synthase identified from $P$. fluorescens KLR101 had high sequence similarity with type II PHA synthase PhaC2. However, the synthase was found to be different owing to its unique gene structure and much higher substrate specificity for scl-PHA precursors over mcl-PHA precursors. Therefore, the PHA synthase from P. fluorescens KLR101 could be categorized as a type I synthase.

\section{Acknowledgments}

We would like to thank Dr. Jaeho Cha (Pusan National University) for his constructive comments. This work was supported by a National Research Foundation of Korea (NRF) grant funded by the Korea government (Ministry of Science, ICT \& Future Planning) (No. NRF-2017R1C1B2003286).

\section{Conflict of Interest}

The authors have no financial conflicts of interest to declare.

\section{References}

1. Anderson AJ, Dawes EA. 1990. Occurrence, metabolism, metabolic role, and industrial uses of bacterial polyhydroxyalkanoates. Microbiol. Rev. 54: 450-472.

2. Madison LL, Huisman GW. 1999. Metabolic engineering of poly(3-hydroxyalkanoates): from DNA to plastic. Microbiol. Mol. Biol. Rev. 63: 21-53.

3. Peoples OP, Sinskey AJ. 1989. Poly-beta-hydroxybutyrate (PHB) biosynthesis in Alcaligenes eutrophus H16. Identification and characterization of the PHB polymerase gene ( $p h b C)$. J. Biol. Chem. 264: 15298-15303.

4. Schubert P, Steinbuchel A, Schlegel HG. 1988. Cloning of the Alcaligenes eutrophus genes for synthesis of poly-betahydroxybutyric acid (PHB) and synthesis of PHB in Escherichia coli. J. Bacteriol. 170: 5837-5847.

5. Slater SC, Voige WH, Dennis DE. 1988. Cloning and expression in Escherichia coli of the Alcaligenes eutrophus H16 poly-beta-hydroxybutyrate biosynthetic pathway. J. Bacteriol. 170: 4431-4436.

6. Kadouri D, Jurkevitch E, Okon Y, Castro-Sowinski S. 2005. Ecological and agricultural significance of bacterial polyhydroxyalkanoates. Crit. Rev. Microbiol. 31: 55-67.

7. Rehm BH, Steinbuchel A. 1999. Biochemical and genetic analysis of PHA synthases and other proteins required for PHA synthesis. Int. J. Biol. Macromol. 25: 3-19.

8. Lee HJ, Choi MH, Kim TU, Yoon SC. 2001. Accumulation of polyhydroxyalkanoic acid containing large amounts of unsaturated monomers in Pseudomonas fluorescens BM07 utilizing saccharides and its inhibition by 2-bromooctanoic acid. Appl. Environ. Microbiol. 67: 4963-4974.

9. Sheu DS, Wang YT, Lee CY. 2000. Rapid detection of polyhydroxyalkanoate-accumulating bacteria isolated from the environment by colony PCR. Microbiology 146: 2019-2025.

10. Solaiman DK, Ashby RD, Foglia TA. 2000. Rapid and specific identification of medium-chain-length polyhydroxyalkanoate synthase gene by polymerase chain reaction. Appl. Microbiol. Biotechnol. 53: 690-694.

11. Timm A, Steinbuchel A. 1990. Formation of polyesters consisting of medium-chain-length 3-hydroxyalkanoic acids from gluconate by Pseudomonas aeruginosa and other fluorescent pseudomonads. Appl. Environ. Microbiol. 56: 3360-3367.

12. Tobin KM, O'Connor KE. 2005. Polyhydroxyalkanoate accumulating diversity of Pseudomonas species utilising aromatic hydrocarbons. FEMS Microbiol. Lett. 253: 111-118.

13. Thompson JD, Gibson TJ, Plewniak F, Jeanmougin F, Higgins DG. 1997. The CLUSTAL_X Windows interface: flexible strategies for multiple sequence alignment aided by quality analysis tools. Nucleic Acids Res. 25: 4876-4882.

14. Kumar S, Tamura K, Nei M. 2004. MEGA3: integrated software for molecular evolutionary genetics analysis and sequence alignment. Brief. Bioinform. 5: 150-163.

15. Hall B, Baldwin J, Rhie HG, Dennis D. 1998. Cloning of the Nocardia corallina polyhydroxyalkanoate synthase gene and production of poly-(3-hydroxybutyrate-co-3-hydroxyhexanoate) and poly-(3-hydroxyvalerate-co-3-hydroxyheptanoate). Can. J. Microbiol. 44: 687-691.

16. Kalousek S, Lubitz W. 1995. High-level poly(beta-hydroxybutyrate) production in recombinant Escherichia coli in sugarfree, complex medium. Can. J. Microbiol. 41(Suppl 1): 216-221.

17. Zorn H, Breithaupt DE, Takenberg M, Schwack W, Berger RG. 2003. Enzymatic hydrolysis of carotenoid esters of marigold flowers (Tagetes erecta L.) and red paprika (Capsicum annuum L.) by commercial lipases and Pleurotus sapidus extracellular lipase. Enzyme Microb. Technol. 32: 623-628.

18. Qi Q, Steinbuchel A, Rehm BH. 1998. Metabolic routing towards polyhydroxyalkanoic acid synthesis in recombinant Escherichia coli (fadR): inhibition of fatty acid beta-oxidation by acrylic acid. FEMS Microbiol. Lett. 167: 89-94.

19. DiRusso CC, Nunn WD. 1985. Cloning and characterization of a gene $(\mathrm{fadR})$ involved in regulation of fatty acid 
metabolism in Escherichia coli. J. Bacteriol. 161: 583-588.

20. Ostle AG, Holt JG. 1982. Nile blue A as a fluorescent stain for poly-beta-hydroxybutyrate. Appl. Environ. Microbiol. 44: 238-241.

21. Brandl H, Gross RA, Lenz RW, Fuller RC. 1988. Pseudomonas oleovorans as a source of poly(beta-hydroxyalkanoates) for potential applications as biodegradable polyesters. Appl. Environ. Microbiol. 54: 1977-1982.

22. Qi Q, Steinbuchel A, Rehm BHA. 2000. In vitro synthesis of poly(3-hydroxydecanoate): purification and enzymatic characterization of type II polyhydroxyalkanoate synthases PhaC1 and PhaC2 from Pseudomonas aeruginosa. Appl. Microbiol. Biotechnol. 54: 37-43.

23. Ellman GL. 1959. Tissue sulfhydryl groups. Arch. Biochem Biophys. 82: 70-77.

24. Gerngross TU, Martin DP. 1995. Enzyme-catalyzed synthesis of poly[(R)-(-)-3-hydroxybutyrate]: formation of macroscopic granules in vitro. Proc. Natl. Acad. Sci. USA 92: 6279-6283.

25. Gerngross TU, Snell KD, Peoples OP, Sinskey AJ, Csuhai E, Masamune S, et al. 1994. Overexpression and purification of the soluble polyhydroxyalkanoate synthase from Alcaligenes eutrophus: evidence for a required posttranslational modification for catalytic activity. Biochemistry 33: 9311-9320.

26. Kraak MN, Kessler B, Witholt B. 1997. In vitro activities of granule-bound poly[(R)-3-hydroxyalkanoate]polymerase C1 of Pseudomonas oleovorans - development of an activity test for medium-chain-length-poly(3-hydroxyalkanoate) polymerases. Eur. J. Biochem. 250: 432-439

27. Huisman GW, Deleeuw O, Eggink G, Witholt B. 1989. Synthesis of poly-3-hydroxyalkanoates is a common feature of fluorescent pseudomonads. Appl. Environ. Microbiol. 55: 1949-1954.

28. Shine J, Dalgarno L. 1975. Determinant of cistron specificity in bacterial ribosomes. Nature 254: 34-38.

29. Dixon R. 1986. The xylABC promoter from the Pseudomonas putida Tol plasmid is activated by nitrogen regulatory genes in Escherichia coli. Mol. Gen. Genet. 203: 129-136.

30. Huisman GW, Wonink E, Meima R, Kazemier B, Terpstra P, Witholt B. 1991. Metabolism of poly(3-hydroxyalkanoates)
(PHAs) by Pseudomonas oleovorans. Identification and sequences of genes and function of the encoded proteins in the synthesis and degradation of PHA. J. Biol. Chem. 266: 2191-2198.

31. Song J, Jensen RA. 1996. PhhR, a divergently transcribed activator of the phenylalanine hydroxylase gene cluster of Pseudomonas aeruginosa. Mol. Microbiol. 22: 497-507.

32. Ciesielski S, Cydzik-Kwiatkowska A, Pokoj T, Klimiuk E. 2006. Molecular detection and diversity of medium-chainlength polyhydroxyalkanoates-producing bacteria enriched from activated sludge. J. Appl. Microbiol. 101: 190-199.

33. Matsusaki H, Manji S, Taguchi K, Kato M, Fukui T, Doi Y. 1998. Cloning and molecular analysis of the poly(3-hydroxybutyrate) and poly(3-hydroxybutyrate-co-3-hydroxyalkanoate) biosynthesis genes in Pseudomonas sp. strain 61-3. J. Bacteriol. 180: 6459-6467.

34. Nishikawa T, Ogawa K, Kohda R, Wang ZX, Miyasaka H, Umeda F, et al. 2002. Cloning and molecular analysis of poly(3-hydroxyalkanoate) biosynthesis genes in Pseudomonas aureofaciens. Curr. Microbiol. 44: 132-135.

35. Timm A, Steinbuchel A. 1992. Cloning and molecular analysis of the poly(3-hydroxyalkanoic acid) gene locus of Pseudomonas aeruginosa PAO1. Eur. J. Biochem. 209: 15-30.

36. Saitou N, Nei M. 1987. The neighbor-joining method - a new method for reconstructing phylogenetic trees. Mol. Biol. Evol. 4: 406-425.

37. Rhie HG, Dennis D. 1995. Role of $f a d R$ and atoC (Con) mutations in poly(3-hydroxybutyrate-co-3-hydroxyvalerate) synthesis in recombinant pha(+) Escherichia coli. Appl. Environ Microbiol. 61: 2487-2492.

38. Brosius J. 1988. Expression vectors employing lambda-, trp-, lac-, and lpp-derived promoters. Biotechnology 10: 205-225.

39. Tabor S, Richardson CC. 1985. A bacteriophage T7 RNA polymerase/promoter system for controlled exclusive expression of specific genes. Proc. Natl. Acad. Sci. USA 82: 1074-1078.

40. Fitch WM. 1971. Toward defining the course of evolution: minimum change for a specific tree topology. Syst. Zool. 20: 406-416. 